Service social

\title{
La prévention du suicide chez les jeunes LGBT accueillis au Refuge en France
}

\section{Isabelle Chollet}

Volume 59, numéro 1, 2013

L'homophobie et les tentatives de suicide et la résilience chez les jeunes LGBTQ

URI : https://id.erudit.org/iderudit/1017480ar

DOI : https://doi.org/10.7202/1017480ar

Aller au sommaire du numéro

Éditeur(s)

École de service social de l’Université Laval

ISSN

1708-1734 (numérique)

Découvrir la revue

Citer cet article

Chollet, I. (2013). La prévention du suicide chez les jeunes LGBT accueillis au Refuge en France. Service social, 59(1), 81-94. https://doi.org/10.7202/1017480ar d'utilisation que vous pouvez consulter en ligne.

https://apropos.erudit.org/fr/usagers/politique-dutilisation/ 


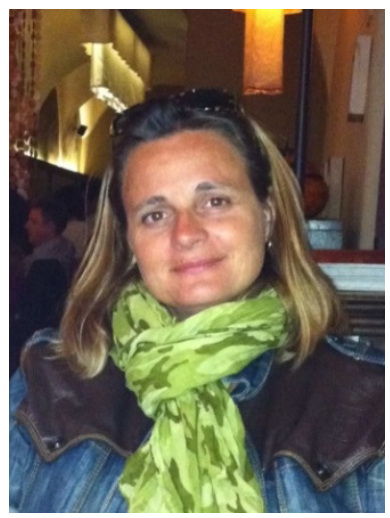

\title{
La prévention du suicide chez les jeunes LGBT accueillis au Refuge en France
}

\author{
par Isabelle CHOLLET, psychologue au Refuge et en cabinet \\ privé. Formatrice à l'Institut Régional de Travail Social du \\ Languedoc Roussillon. ichollet psychologue@yahoo.fr I \\ www.psychologue-nimes-montpellier.com
}

Psychologue depuis plusieurs années auprès de jeunes gays et lesbiennes dans une association qui les accueille lorsqu'ils ont été jetés à la rue ou lorsqu'ils sont victimes d'homophobie, je me suis intéressée à ce qu'ils pouvaient vivre et surtout à ce qu'ils pouvaient attendre des aidants gravitant autour d'eux.

En 2009, lors de la rédaction de mon mémoire en Thérapie par le Jeu et la Créativité, j'ai souhaité réfléchir sur la façon d'aider des thérapeutes à accompagner des personnes pouvant trouver difficile d'assumer leur l'homosexualité difficile. La question du suicide y était évidemment abordée. Pour cela, j'ai réalisé un questionnaire qui devait donner une photographie de la situation, questionnaire qui a eu beaucoup de succès puisque 508 personnes ont accepté d'y répondre sur le site internet du Refuge. Devant un tel engouement, il ne m'était pas possible de traiter les résultats et j'ai sollicité Michel Dorais qui a bien voulu reprendre le projet qui a abouti en février 2012 à la parution de Être homo aujourd'hui en France (Édition H\&O). Je précise juste que ce questionnaire proposait des questions ouvertes et fermées permettant de mettre au jour ce que je pouvais entendre régulièrement dans mon cabinet.

Dans ma conférence à Trois-Rivières, j'ai dans une première partie présenté le public qui a répondu au questionnaire puis j'ai expliqué ce qu'est le Refuge en France et son activité, avant d'orienter mon propos plus précisément sur les accompagnements psychologiques et sur quelques suggestions quant à la question de la prévention du suicide.

En guise de préambule, je tenais à donner la parole à des jeunes que j'ai pu accueillir dans mon cabinet pouvant me parler d'une manière ou d'une autre du suicide et du mal-être afin que vous puissiez évaluer la détresse et la violence des propos.

Quelques mots autour du suicide...

"Quand je l'ai dit à ma mère, elle m'a répondu que tout le monde pouvait changer, que c'était possible, qu'il me fallait combattre le sheitan [diable, en arabe]. Quand je lui ai dit que c'était impossible, elle m'a répondu que si c'était ce que je souhaitais, je ne pouvais plus vivre chez eux, que ce n'était pas compatible avec leurs convictions religieuses et qu'il me fallait quitter le domicile familial. Quand je 
suis sorti de la maison, je leur ai dit que je les aimerais toujours et la dernière chose que j'ai entendue après avoir passé la porte a été : crève vite. »

«Tu n'as jamais pensé à te suicider? Ça soulagerait ta mère... » (propos du beaupère).

"J'ai la chance d'avoir de bonnes relations avec mes parents mais je me sens détruit par les insultes homophobes. J'ai fait une TS [tentative de suicide] car j'en voulais à tous ceux qui ne me laissaient pas être ce que je suis. Je me suis scarifié pour montrer ma colère et que j'en ai marre. $Y$ a qu'une personne qui a le droit de me faire souffrir, c'est moi! »

« Je me violentais pour ne pas violenter les autres. La souffrance, elle est pire que la mort. »

«C'est important pour moi d'être au Refuge : c'est rassurant, reposant, et je suis entouré de jeunes comme moi »

Pour tenter d'évaluer les besoins en termes thérapeutiques (car tel était mon objectif de départ), j'ai donc lancé cette enquête en 2009, à laquelle 508 personnes ont répondu, dont $72 \%$ d'hommes et $28 \%$ de femmes. Michel Dorais a pris le relais et coordonné la suite de l'enquête grâce à une subvention attribuée par l'Université Laval.

Pour continuer sur le profil des répondants, $38 \%$ avaient entre 15 et 24 ans, $26 \%$ entre 25 et 34 ans et $36 \%$ plus de 35 ans. L'âge médian était de 28,5 ans. Les personnes interrogées provenaient à $76 \%$ de grandes villes et $28 \%$ se déclaraient croyants.

Ce que cette enquête a notamment révélé, c'est que l'on découvrait maintenant sa sexualité plus tôt, à 15 ans (plutôt qu'à 17 ans en 1984, date du « Rapport Gai »), alors que la révélation aux parents serait plus tardive, à 20-22 ans (plutôt qu'à 18-19 ans en 1984). On vivrait donc aujourd'hui cette réalité plus longtemps dans la solitude. Le coming-out par ailleurs serait un risque calculé, sélectif et successif (parents, famille, amis, collègues...).

Ce travail de recherche montre notamment que la peur des autres reste très présente: l'homophobie serait vue comme responsable de la honte et de la peur de s'affirmer. Quant au sujet qui nous intéresse particulièrement, notons que $30 \%$ des répondants disent avoir fait une tentative de suicide (environ 10 fois plus que la population générale).

Pour enrayer ce fléau, pour comprendre et soutenir ces personnes souvent jeunes qui veulent mettre fin à leurs jours, une question leur a notamment été posée sur la communauté gay et le soutien qu'elles pouvaient en attendre. On s'aperçoit qu'à peine plus de $30 \%$ des répondants ont fait ou font partie d'une association homosexuelle mais que le milieu gay ou lesbien et leurs associations sont un soutien pour assumer positivement son homosexualité. Toutefois, les critiques du milieu gay portent sur son manque d'ouverture et de solidarité. Beaucoup de jeunes accueillis au Refuge parlent de l'importance de partager cette situation avec d'autres. 
Dans ce travail, nous avons également interrogé les personnes sur la vie de couple et la vie de famille. $60 \%$ ont vécu ou vivent en couple de façon « officielle » (contre $36 \%$ des hommes et $56 \%$ des femmes en 1984). Par ailleurs, près de $60 \%$ souhaitent avoir des enfants (50 \% des hommes, < $25 \%$ des femmes en 1984). Le projet de loi pour instituer le «mariage pour tous » demeure très controversé. Les manifestations de ces dernières semaines nous montrent bien qu'en France, la légalisation est loin d'être acquise comme au Québec.

L'important pour moi était de connaître les difficultés des répondants et leurs attentes en termes de thérapie, thérapie à laquelle beaucoup ont eu recours puisque $47 \%$ ont consulté un psychologue ou un psychothérapeute. Ce qui a été clairement exprimé dans les réponses, c'est qu'à $71 \%$ les répondants n'attendaient pas de leur thérapeute qu'il soit homosexuel comme eux, mais qu'il ait une bonne connaissance des réalités LGBT et qu'il y soit sensible. Soit dit en passant, même s'il ne semble pas exister de structures similaires au Refuge au Québec, la formation des thérapeutes professionnels y est beaucoup plus développée, et ce, depuis bien des années.

Abordons maintenant les principales difficultés psychologiques de cette population particulièrement à risque. Ce qui est très présent dans les difficultés exprimées, c'est de s'accepter, de s'estimer. Beaucoup de honte et de culpabilité demeure associées à I'homosexualité, ce qui entraîne en conséquence l'isolement, la solitude et le repli sur soi. La peur du regard, du jugement des autres, la peur du rejet, la peur de décevoir est présente également. Alors, comment s'accepter en ayant ces sentiments de culpabilité, de honte, de peur, de mauvaise estime de soi ?

Beaucoup de temps sera nécessaire au jeune pour accepter qui il est sans l'avoir choisi (pour $88 \%$ des répondants, l'homosexualité ne serait pas un choix). On estime ce temps de silence à entre cinq et neuf ans. Socialement, le jeune se sentira donc isolé, incompris, en danger à cause des actes homophobes. II pourra même se sentir de trop: ce qui pourra l'amener au suicide.

C'est devant le besoin évident de lutter contre l'homophobie, contre l'isolement, contre les difficultés associées au fait de vivre sa vie homosexuelle, que le Refuge est né il y a tout juste dix ans. Étonnée que ce type de structure n'existe pas au Québec, je vais vous présenter les missions et le fonctionnement de cette association unique en France et inexistante de l'autre côté de l'Atlantique.

Le Refuge a été créé en janvier 2003 par Nicolas Noguier (actuel président) pour accueillir des jeunes de 18 à 25 ans, filles et garçons, en situation d'isolement du fait de leur attirance pour une personne de même sexe. Huit années d'interventions énergiques ont été nécessaires pour que cette association soit reconnue d'utilité publique.

Le siège de l'association se situe à Montpellier. Cinq délégations sont présentes sur le territoire français: Montpellier, Paris, Marseille, Lyon et Toulouse. Un total de 1103 adhérents sont plus ou moins actifs dans son fonctionnement. Une centaine de bénévoles s'impliquent audelà d'une aide financière et garantissent environ 7000 heures d'intervention. Le budget de 
fonctionnement est de $457000 €$ soit environ 570000 \$ canadiens. La vocation de l'Association est de prévenir et de rompre l'isolement en proposant de l'hébergement temporaire, un accompagnement social et psychologique, une écoute et de la médiation familiale, des interventions en milieu scolaire.

Depuis 2003, 1418 jeunes sont entrés en contact avec l'association, 182 jeunes ont été hébergés, 86 ont été accompagnés à distance et réorientés, 80 ont été accompagnés en accueil de jour hors hébergement.

Les trois axes prioritaires de l'action du Refuge en 2011 ont été : (1) l'information et l'intervention dans les établissements scolaires afin de sensibiliser les jeunes adolescents à la problématique de l'homophobie; (2) la promotion de la prévention des pratiques à risques, notamment dans la lutte contre le VIH, les IST [infections sexuellement transmissibles] et les MST [maladies sexuellement transmissibles] ; et enfin (3) la lutte contre le suicide.

L'association dispose de lieux pour recevoir les jeunes en hébergement à Montpellier, Paris et Lyon. Dans d'autres villes, nous ne pouvons proposer à ce jour que des espaces de rencontre et des permanences (Marseille, Narbonne, Saint-Denis de la Réunion...). Nous offrons également un service d'écoute téléphonique avec le numéro d'urgence du Refuge (accessible aux mineurs), un service d'échange via courriels ou textos et enfin des interventions de terrain (sensibilisation/formation).

Nous sommes une équipe de six salariés : trois conseillères en économie familiale et sociale à Montpellier, Paris et Marseille, un directeur général (Frédéric Gal), un secrétaire (Paris), un chargé de mission et des vacataires (psychologues à Marseille et Montpellier). À cela s'ajoutent une équipe de 100 bénévoles (7000 heures), huit volontaires du service civique (contrat de six mois) et, en moyenne, 15 stagiaires (travailleurs sociaux).

En ce qui concerne notre financement, $37 \%$ proviennent de dons et cotisations, $22 \%$ de collectivités territoriales (mairies, conseils généraux, régions), $28 \%$ de l'État, $10 \%$ du mécénat, $3 \%$ d'autres sources (ventes et produits divers).

En 2011, 80 jeunes ont profité d'un hébergement (dont $20 \%$ de filles) pour 479 demandes. Le jeune à l'arrivée va signer un contrat d'accompagnement et d'hébergement, puis s'installer dans un des appartements (souvent logés à deux pour éviter l'isolement). II aura également accès aux soins (médecin, psychologue, dentiste, au besoin), car certains jeunes ne sont pas allés consulter pendant longtemps du fait de leur situation d'errance. On mettra en place un accompagnement social (démarches pour l'obtention de papiers, mise en disposition de se former ou de travailler, accès au logement). Le jeune devra également rencontrer le psychologue de l'association pour évaluer le rythme des rencontres. II sera également dans l'obligation de participer aux permanences qui ont lieu trois fois par semaine. Il lui sera proposé des ateliers de CV, de recherche d'emploi, ainsi que des activités sportives et culturelles.

La durée d'accompagnement totale est de six mois, laquelle pourra être réduite ou étendue selon les besoins et les choix du jeune. 


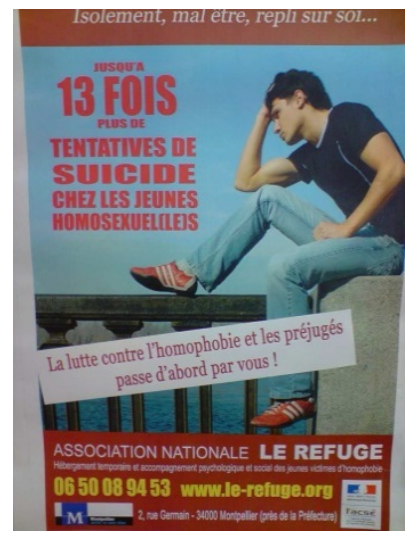

Au-delà de la mission d'accompagnement des jeunes, l'une des grandes missions du Refuge est de lutter contre l'homophobie. Pour cela, des campagnes de prévention sont régulièrement organisées. L'association va informer de ses activités. Elle rendra accessible les informations pour les personnes isolées ou loin des lieux de permanence. Elle favorisera le soutien psychologique. Son approche est d'occuper le terrain pour toucher, informer et prévenir. Des campagnes publicitaires sont régulièrement organisées et certaines villes de France, voire certains lieux partenaires comme des cliniques, en permettent

l'affichage. L'association fait en sorte d'être également très présente sur la Toile par son site internet (580 visites par jour en moyenne : http://www.le-refuge.org), un groupe Facebook « Le Refuge: contre le mal-être des jeunes victimes d'homophobie et de transphobie » (4500 membres) ainsi qu'un compte Twitter. Les portes de l'association sont régulièrement ouvertes aux médias : interventions TV, radio, journaux. Sont invitées également le plus souvent possible des personnalités politiques. L'association participe aussi aux différentes manifestations de lutte contre l'homophobie et les IST, et elle intervient, comme on l'a dit, dans les établissements scolaires (agrément de l'Éducation Nationale). Elle essaye de promouvoir la recherche et l'édition d'ouvrages.
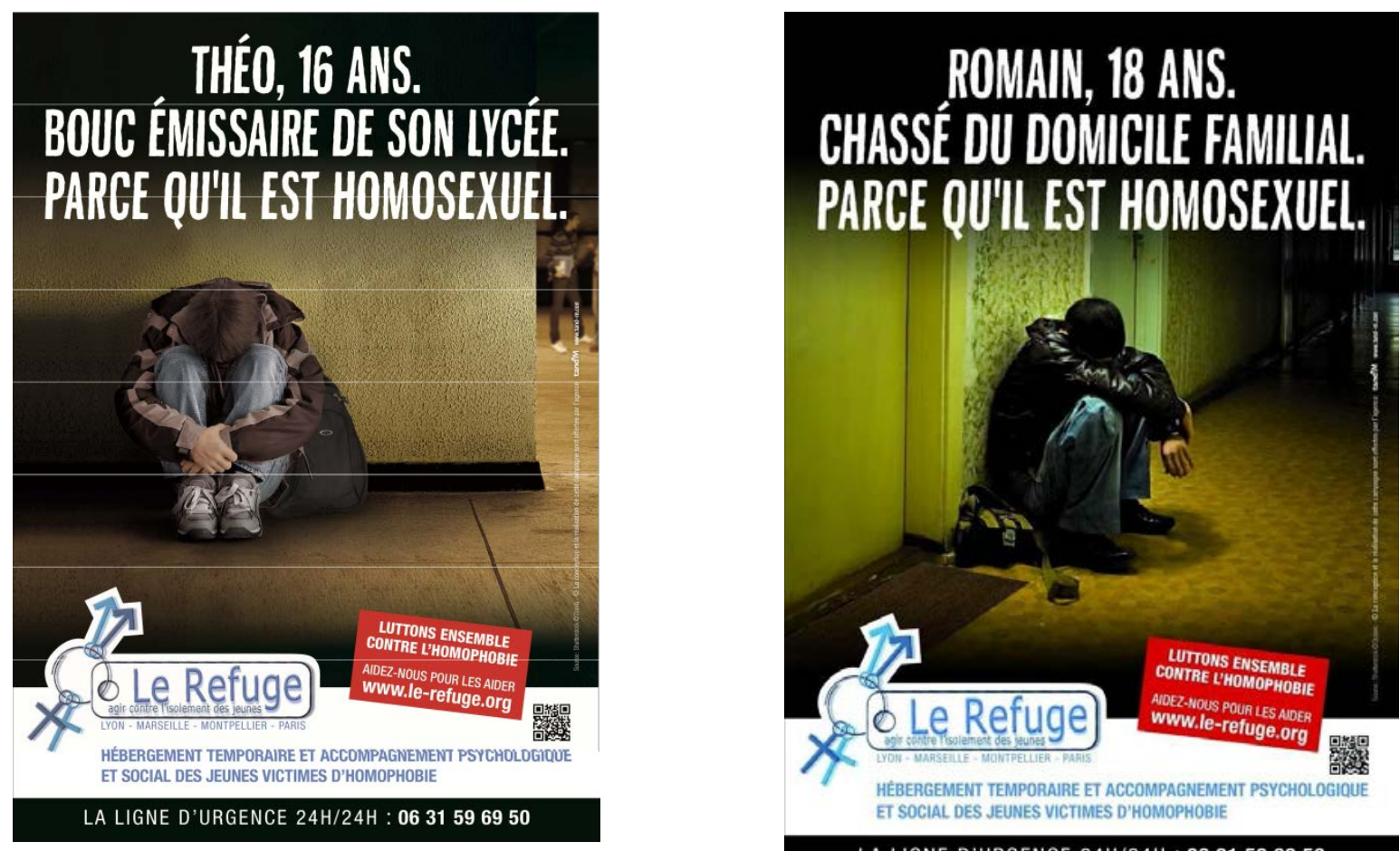

LA LIGNE D'URGENCE 24H/24H : 0631596950 
Focalisons-nous plus précisément sur les accompagnements psychologiques. Un premier rendez-vous est prévu pour rencontrer le jeune, évaluer ses besoins, déterminer s'il a besoin d'un suivi hebdomadaire ou mensuel, d'une rencontre avec un médecin psychiatre par l'intervention de I'UMIPP [Unité mobile intersectorielle de psychiatrie pour populations en situation de précarité]. Ce travail en partenariat avec un service psychiatrique peut être d'une aide précieuse car les jeunes quelquefois en situation de dépression peuvent avoir besoin d'un soutien médicamenteux pour éviter le passage à l'acte.

En ce qui concerne les attentes générales révélées par l'enquête mais également par les jeunes du Refuge, les personnes ne viennent pas consulter sur le pourquoi elles sont homosexuelles mais sur le comment vivre avec cette réalité. Elles ont besoin de se sentir en sécurité pour aborder des choses qu'elles n'ont parfois jamais exprimées. Un climat de confiance et de confidentialité sont essentiels. La grande solitude dans laquelle elles se sont trouvées pour la plupart, à ne pouvoir parler à personne de ce qui les envahissait, les font avoir besoin d'une interaction importante avec le thérapeute, voire de solliciter des conseils. Les qualités qu'elles mentionnent le plus souhaiter chez leur thérapeute serait l'« homosensibilité », l'écoute, l'ouverture, la compétence et la compréhension, la présence, l'empathie, la capacité à rassurer.

Les difficultés les plus susceptibles de mener à l'envie d'en finir seraient liées à de grandes souffrances familiales, à des troubles d'attachement (mal attaché/détaché : la solitude peut en effet pousser ces jeunes à s'attacher très vite pour se retrouver à nouveau très vite dans une situation de rupture et d'isolement). Beaucoup de jeunes du Refuge ont également évoqué une grande fragilité liée à la violence de l'exclusion subie (séparation de la famille, des amis, du quartier), à de la maltraitance physique ou psychologique (rarement sexuelle), aux propos homophobes encaissés à répétition depuis des années. Une réelle souffrance d'être homosexuel(le) alors qu'on ne l'a pas choisi est perceptible. L'insupportable et pour certains l'insurmontable vont favoriser des postures d'addiction, de prise de risque, voire de prostitution, quand ces jeunes exclus se sont retrouvés dans la rue sans ressources. Les difficultés psychologiques ne sont évidemment pas liées exclusivement à l'homosexualité, les choses étant imbriquées les unes dans les autres.

Pour aborder les histoires de vie et les souffrances de ces jeunes, je leur ai proposé la Thérapie par le Jeu et la Créativité, qui leur permet de développer créativement des ressources pour survivre. Cette approche thérapeutique individuelle, développée par Verity J. Gavin (britannique et française), se situe à la croisée de plusieurs courants (Winnicott, play therapy, thérapie existentielle, neurosciences); elle vise à développer le potentiel créatif de la personne dans sa relation à soi et à l'autre (le thérapeute) pour lui permettre de trouver le courage d'oser s'exprimer et donc d'exister (au regard de soi, puis de l'autre, puis des autres). 


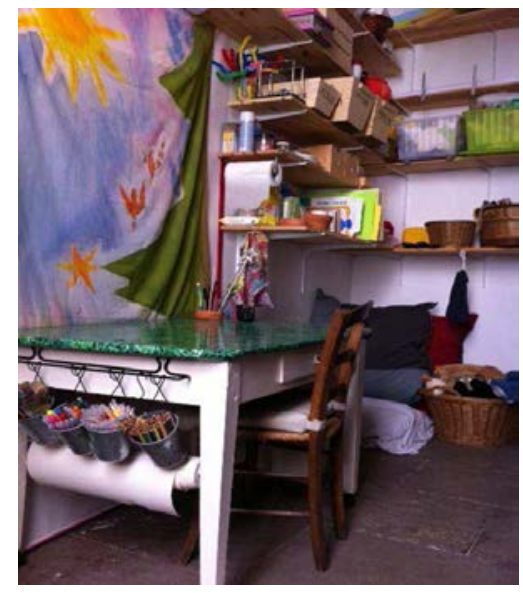

Pour favoriser ce travail, mon cabinet-atelier offre un certain nombre de médias créatifs. La personne aura le choix de s'exprimer oralement ou autrement: modelage, peinture (debout, assis, par terre), marionnettes... L'important est d'offrir de larges propositions qui seront saisies ou pas par le patient, selon l'envie ou le moment. Le but de cette approche est d'explorer pour oser se montrer comme on est et s'accepter, le tout dans un cadre sécurisant et bienveillant. II s'agit pour le patient de s'appuyer sur ses potentialités pour oser regarder la partie plus sombre de son être. Le travail se fait avec les enfants, les adolescents ou les adultes.

Il me paraît important de décrire quelques exemples de travail avec la Thérapie par le Jeu et la Création en cabinet-atelier pour évaluer l'intérêt que pourrait avoir cette approche dans la prévention du suicide. Par les exemples ci-dessous, je montrerai comment on peut être amené à exprimer des choses qui ne « sortiraient » pas autrement.

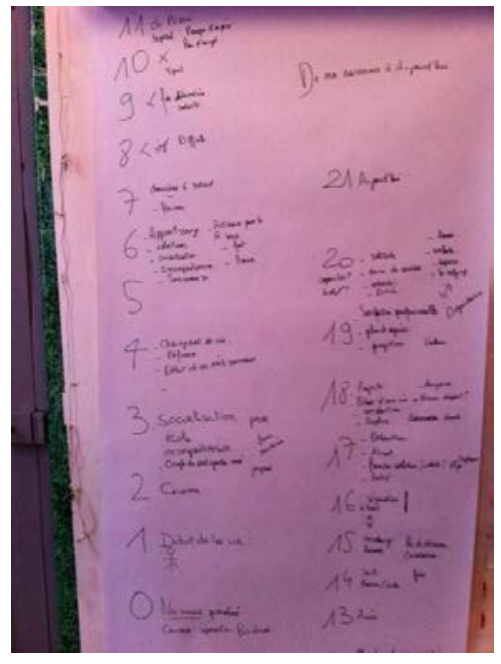

Parler de sa vie déjà peut permettre aux personnes d'avoir accès à des souvenirs qu'elles vont resituer dans le temps. Ici, la personne va corporellement bouger pour écrire les différents événements de vie de façon chronologique sur une affiche de $2 \mathrm{~m}$ de hauteur de manière à dessiner debout et inclure une dimension corporelle faisant leur hauteur. Se remémorer, écrire, dire, faire des liens, évaluer le laps de temps où les choses ont été difficiles ou pas. 


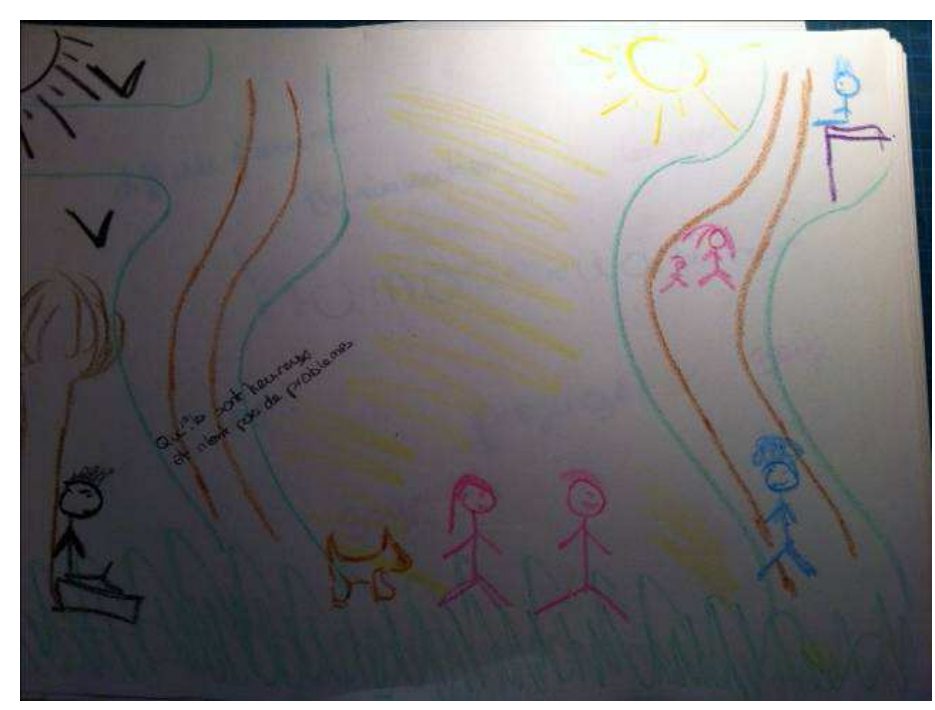

«Une pierre pour me représenter »
Autre exemple, ici, le jeune que je vois pour la deuxième fois va exprimer l'état de solitude dans lequel il se sent. Même si le dessin paraît enfantin (les adolescents ou les adultes que je reçois ne sont pas des dessinateurs), ce qu'il est important de noter ici n'est pas la qualité artistique mais bien la sensation dans laquelle le patient se trouve. Ici, le jeune à gauche se représente tout seul adossé à un arbre. Tout autour de lui est noir. II se dessine en noir. II est triste et malheureux. II dessine même un soleil noir alors qu'à droite, il dessine des gens qu'il ne connaît pas avec des chiens. II dessine des gens qui sont heureux et qui n'ont pas de problème. II explique, lui, qu'il a un gros problème. Il est homosexuel!

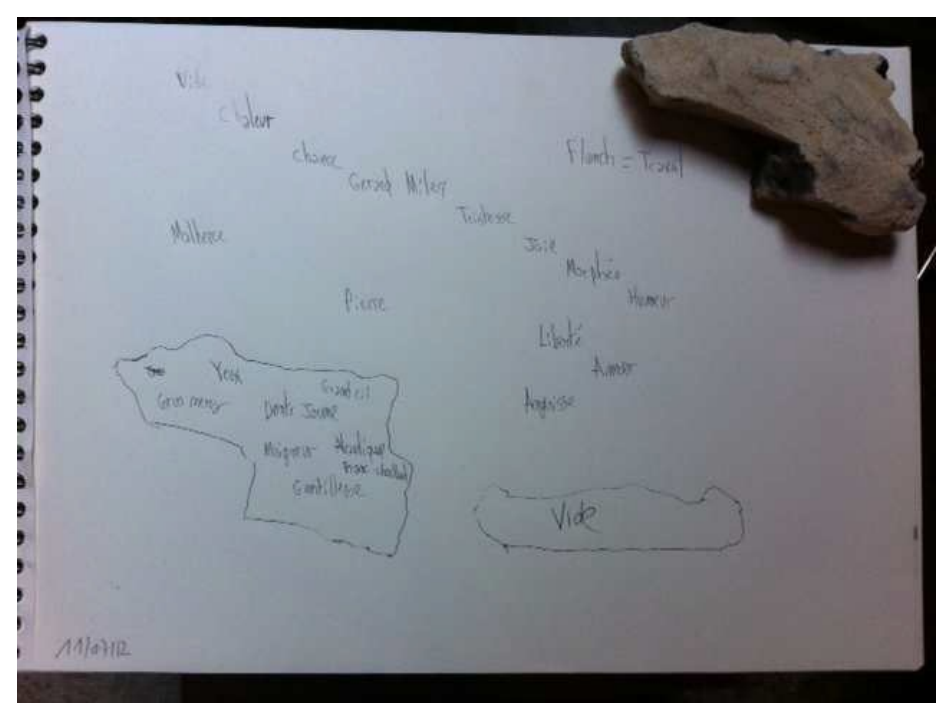

Ici, je reçois un jeune qui n'arrive absolument pas à parler de lui, à s'ouvrir alors qu'il fait beaucoup d'insomnies, qu'il n'est pas bien.

Je lui ai demandé ici de choisir une pierre qui pouvait le représenter. Comme cela lui paraissait assez peu sérieux, il a commencé, avec beaucoup de sérieux justement, à en choisir une. II m'a ensuite expliqué pourquoi ce choix: une pierre claire à l'extérieure et noire à l'intérieur. Sur un bloc $A 3$, il a ensuite tracé le contour de la pierre en inscrivant tout ce qui pouvait le qualifier à l'extérieur. Puis, il a dessiné la pierre sur la tranche pour y inscrire ce qui pouvait le caractériser à l'intérieur et là, il a écrit « vide ». 


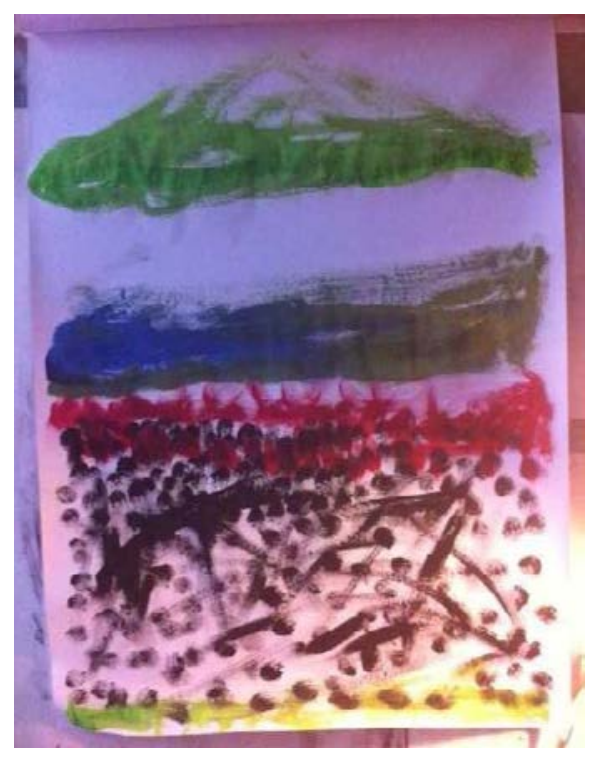

« Des couleurs pour traduire ce que je ressens »

Un autre exemple d'une jeune femme vraiment pas bien. Lorsqu'elle est arrivée, elle ne voulait rien dire, elle n'arrivait pas à parler, à exprimer l'état dans lequel elle se trouvait. Je lui ai donc demandé de dessiner comment elle se sentait et d'écrire ce qui lui venait.

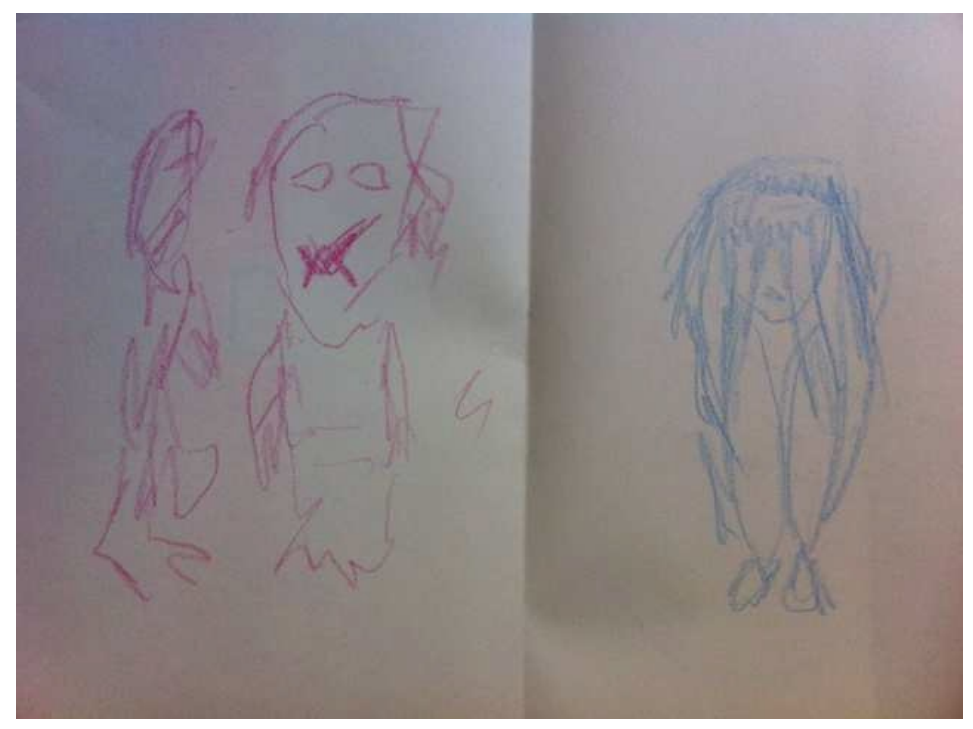

Suite à ce dessin, elle a écrit qu'elle se sentait mal à l'aise, gênée, isolée, qu'elle manquait de confiance et qu'elle avait peur d'être qui elle était. Cette consultation a été l'une des premières où elle a pu admettre ce qui était difficile, voire insoutenable, pour elle d'être homosexuelle. 


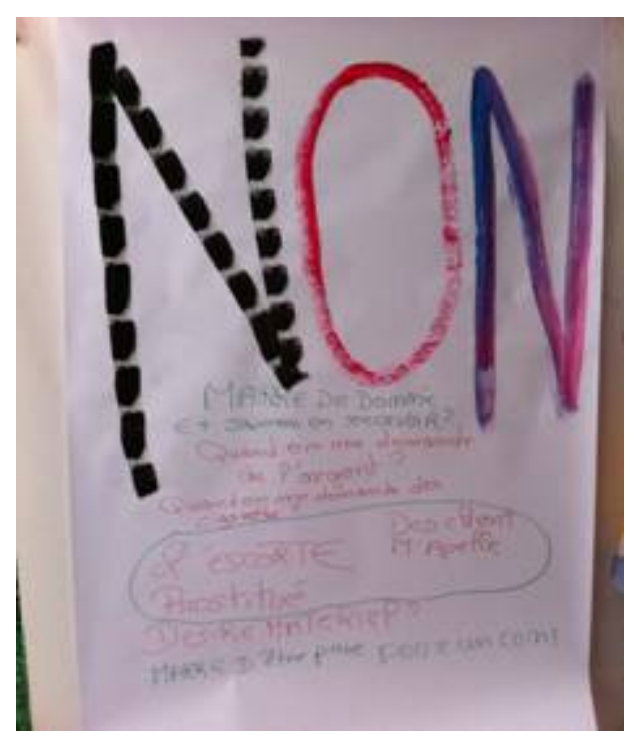

Enfin, pour la dernière image, on a intégré une approche que l'on utilise en Thérapie par le Jeu et la Créativité mais qui existe comme approche thérapeutique à part entière: la thérapie par le jeu de sable [International Society for Sandplay Therapy, ISST] qui est d'ailleurs utilisée au Québec plus qu'en France. Ici, le jeune a créé dans le bac de sable un monde autour de la mort lui permettant d'exprimer son mal-être, de faire apparaître ses vieux démons par la présence d'arbres morts, de deux cercueils dont un orné d'un bébé (lui), de serpents, de squelettes, d'un corbeau. Cette approche, qu'il serait trop long d'expliciter, a permis à ce jeune de mettre en scène une ambiance, avec des figurines qui se retrouvaient dans ses bacs successifs, de matérialiser une situation dont il a pu dire quelque chose après.

\section{Arriver à oser dire « NON »}

Ici, un jeune qui, notamment de par son vécu de prostitué, est dans l'incapacité de dire non.

Comme dans l'exemple plus haut, il a été intéressant pour lui, outre d'exprimer par la parole son souhait d'arriver à dire non, de mettre son corps en mouvement, de manifester sa colère, en y mettant un nouveau sens (du fait de l'intégration d'un nouveau sens : la vue).

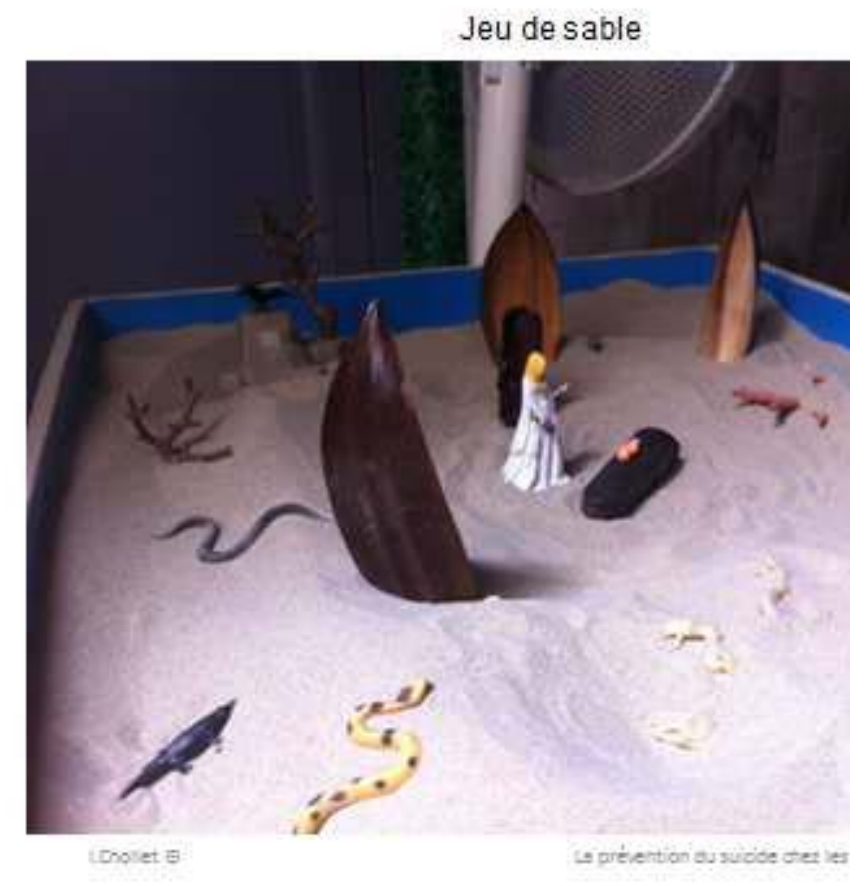


Permettre aux personnes de libérer non seulement leur parole mais d'ouvrir un espace potentiel créatif leur permettant d'explorer en toute sécurité les dimensions de leur être ne suffit toutefois pas : les jeunes du Refuge, comme les répondants de l'enquête ou même les patients que je peux avoir en thérapie par téléphone ou en cabinet, ont besoin d'une ouverture à la diversité sexuelle. Une tentative de suicide est souvent liée au sentiment d'être incompris et de ne pouvoir libérer l'expression de sa différence. La honte, la culpabilité, la peur peuvent mener à percevoir le suicide comme une délivrance vis-à-vis de quelque chose que l'on n'a pas choisi. Les jeunes qui nous arrivent au Refuge sont porteurs d'une histoire difficile semée d'embûches, et le souhait d'en finir est la conséquence d'éléments qui leur échappent souvent.

Confrontée comme je le suis régulièrement à des jeunes tentés par le suicide, il me paraît important de faire quelques suggestions pour essayer de le prévenir. Par ailleurs, j'ai réfléchi, en continuité de cette recherche, à un questionnaire que je pourrais faire passer en début de consultation pour évaluer la dangerosité des situations.

Ce que je préconise évidemment d'abord, c'est d'être dans l'écoute et l'acceptation des jeunes. II faudrait favoriser les espaces de décompression/reconstruction/valorisation. En effet, les jeunes qui nous arrivent ont besoin, après avoir vécu l'exclusion parfois violente de leur famille, après avoir parfois vécu dans la rue, de décompresser, de vivre leur homosexualité qu'ils ont toujours cachée. Seulement, cette période de décompression doit se poursuivre par un temps d'acceptation de soi et de construction d'un avenir professionnel et personnel amenant à l'autonomie. (II faut trouver la bonne mesure entre le besoin du jeune d'investir sa vie homosexuelle et celui de se construire un avenir professionnel.)

Il s'agira donc de développer le tissu social et les relations interpersonnelles du patient, lui proposer des échanges individuels et collectifs dans un cadre sécurisant, au sein du Refuge. II sera important de favoriser son ancrage en l'impliquant dans les projets de l'association. II faudrait également multiplier les espaces d'accueil.

Pour les personnes qui ne bénéficient pas d'un hébergement, il faudrait diversifier les moyens de communication et de documentation sur l'homosexualité et le suicide (internet, échanges téléphoniques), et trouver le moyen de rejoindre et accompagner les mineurs, par des initiatives d'aide aux devoirs, par exemple, qui serait la raison officielle des rencontres. II faudrait également favoriser l'échange avec les familles pouvant se trouver également dans la souffrance et dans une forme d'incompréhension.

II me paraît essentiel ensuite de former des bénévoles et des professionnels de l'accompagnement (homophobie/méconnaissance) comme vous pouvez le faire au Canada depuis plus de quinze ans, de travailler en équipe et de faire respecter les contrats des jeunes et des bénévoles.

Pour conclure, je vais vous présenter ce que j'ai pu imaginer et créer comme questionnaire, pour tenter d'évaluer la dangerosité face au suicide. Ce questionnaire pourrait également servir de support d'échange autour de la question de la prévention du suicide. 
Comme la majorité des jeunes hébergés au Refuge ont déjà fait une tentative de suicide, il me paraissait important d'en connaître les circonstances, les causes et, surtout, comment ils avaient trouvé la force de rester vivants et de se présenter dans mon cabinet. J'ai donc imaginé des questions pour connaître la personne, ses facteurs de risque mais surtout ses facteurs de protection ou de résilience. Ce questionnaire est rempli par les jeunes non pas de façon systématique mais lorsqu'il y a des risques. II serait intéressant d'en évaluer les résultats et de les partager avec les chercheurs qui étudient la prévention du suicide.

Isabelle Chollet 


\section{QUESTIONNAIRE SUR LA PRÉVENTION DU SUICIDE (en élaboration)}

\section{Présentation}

$\checkmark$ SEXE : HOMME/FEMME

$\checkmark$ PratiQue RELIGIEUSE FAMILIALE :
ÂGE : ANS

OUI/NON SI OUI, LAQUELLE?

\section{Mon état d'être}

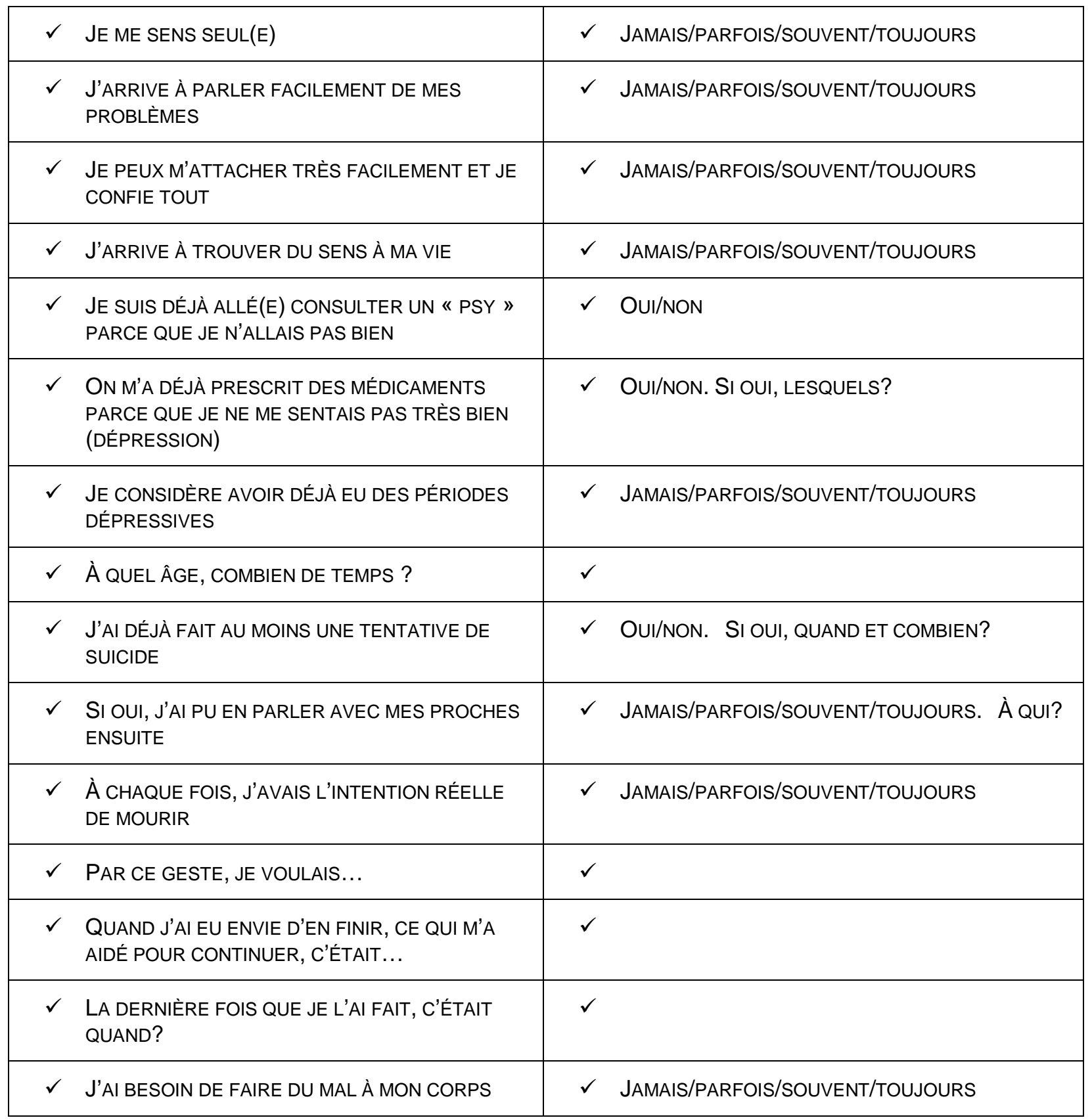




\section{Dire quoi :}

\begin{tabular}{|c|c|c|}
\hline$\checkmark$ JE SUIS QUI J'AI ENVIE D'ÊTRE & $\checkmark$ JAMAIS/PARFOIS/SOUVENT/TOUJOURS \\
\hline$\checkmark \quad$ CE QUE J'AIME CHEZ MOI, C'EST ... & $\checkmark$ \\
\hline$\checkmark \quad$ CE QUE JE N'AIME PAS CHEZ MOI, C'EST $\ldots$ & $\checkmark$ \\
\hline$\checkmark \quad$ J'EXPLIQUE MON MAL-ÊTRE PAR ... & $\checkmark$ \\
\hline$\checkmark \quad$ SI J'AVAIS UNE BAGUETTE MAGIQUE, JE... & $\checkmark$ \\
\hline$\checkmark \quad$ QUAND JE ME SENS MAL, POUR ME SENTIR & $\checkmark$ \\
\hline & MIEUX, JE... & $\checkmark$ \\
\hline
\end{tabular}

\section{L'entourage}

\begin{tabular}{|cl|c|c|}
\hline$\checkmark$ & $\begin{array}{l}\text { J'AI UN PASSÉ FAMILIAL FACILE, NON PLUTÔT } \\
\text { TRĖS }\end{array}$ & $\checkmark$ \\
\hline$\checkmark$ & J'AI DES AMIS À QUI PARLER DE MON MAL-ÊTRE & $\checkmark$ & OUI/NON. SI OUI, COMBIEN? \\
\hline$\checkmark$ & $\begin{array}{l}\text { LES PERSONNES IMPORTANTES POUR MOI } \\
\text { SONT } \ldots\end{array}$ & $\checkmark$ & JAMAIS/PARFOIS/SOUVENT/TOUJOURS \\
\hline$\checkmark$ & JE PEUX LES CONTACTER & $\checkmark$ & JAMAIS/PARFOIS/SOUVENT/TOUJOURS \\
\hline$\checkmark$ & $\begin{array}{l}\text { LES PERSONNES QUE J'AIME PRENNENT DE } \\
\text { MES NOUVELLES }\end{array}$ \\
\hline
\end{tabular}

\section{La vie au Refuge Je suis arrivé(e) au Refuge le.}

\begin{tabular}{|c|c|c|c|}
\hline$\checkmark$ & $\begin{array}{l}\text { JE M'ENTENDS BIEN AVEC LE COLOCATAIRE DE } \\
\text { MON LOGEMENT }\end{array}$ & $\checkmark$ & JAMAIS/PARFOIS/SOUVENT/TOUJOURS \\
\hline$\checkmark \quad \begin{array}{l}\text { AU REFUGE, J'ARRIVE À TROUVER DES } \\
\text { REPĖRES }\end{array}$ & $\checkmark \quad$ JAMAIS/PARFOIS/SOUVENT/TOUJOURS \\
\hline$\checkmark \quad$ JE ME SENS BIEN DANS LE MILIEU HOMOSEXUEL & $\checkmark$ \\
\hline$\checkmark \quad \begin{array}{l}\text { POUR AIDER LES JEUNES QUI ONT DÉJÀ FAIT } \\
\text { DES TENTATIVES DE SUICIDE, JE PENSE QU'IL } \\
\text { FAUDRAIT... }\end{array}$ & $\checkmark$ \\
\hline$\checkmark \quad \begin{array}{l}\text { LES MOMENTS DE LA JOURNÉE QUI ME SONT } \\
\text { LES PLUS DIFFICILES SONT... }\end{array}$ & $\checkmark$ \\
\hline$\quad \begin{array}{l}\text { CE QUI M'AIDE AU REFUGE POUR ME SENTIR } \\
\text { MIEUX, C'EST... }\end{array}$ \\
\hline
\end{tabular}

